\title{
Possible Endpoints in a Prodromal Alzheimer's Disease Trial
}

\author{
A.M. Wessels, S.W. Andersen, P. Castelluccio, Z. Zhang, P. Yu \\ Eli Lilly and Company, Indianapolis, IN, USA \\ Corresponding Author: Alette M Wessels, Eli Lilly and Company, Indianapolis, IN, USA, wesselsal@lilly.com

A lzheimer's Disease (AD) begins with cognitive decline which progresses to affect function, with several analyses demonstrating that cognitive decline precedes and predicts functional decline $(1,2)$. These analyses, based on multiple independent databases, showed that greater cognitive impairment predicts greater functional impairment at the subsequent time point, suggesting that worsening cognition is associated with future functional decline. Thus, a cognitive treatment effect would be expected to lead to a later functional treatment effect. Historically, both a cognitive and a global or functional measure have been used as co-primary outcomes, to demonstrate the effectiveness of AD treatments, in studies of mild to moderate or moderate to severe AD. The global or functional co-primary was required to ensure that a demonstrated cognitive effect was clinically meaningful.

Established instruments to measure cognition and function in subjects with $\mathrm{AD}$ lack sensitivity to assess disease progression and therapeutic response in early stages of the disease process. The registration standard Alzheimer's Disease Assessment Scale Cognitive Subscale (ADAS-Cog) was developed for the moderate stages of $\mathrm{AD}$ and is not sensitive to detect and follow $\mathrm{AD}$ progression during pre-dementia stages (3). In addition, scales to measure activities of daily living were developed to assess function at these later stages and may be less sensitive to early loss.

The US Food and Drug Administration has proposed the possibility of using a composite measure including cognitive and functional elements as a single primary outcome measure in studies of patients with mild cognitive impairment (MCI) due to $\mathrm{AD}$, but the application of this approach may be impractical due to the previously mentioned reasons; i.e., lack of validated cognitive and functional scales that are sensitive to the subtle changes at this stage of the disease.

Despite these challenges and limitations, several efforts have been employed to develop a single scale optimized for MCI due to AD and early AD trials. Those efforts involved the elimination of less informative items from existing cognitive scales and substitution with measures from other instruments of cognition and/or function, with the goal of improving sensitivity to change and reducing variability (4-8). These initiatives used different statistical methodologies and different datasets for the calculation of composites; however, they arrived at largely convergent findings in terms of individual items of existing scales that were found to be sensitive in these early disease stages.

The objectives of this work were to evaluate the performance of various cognitive/functional composites and to compare the performance of each composite against the others and against more commonly used endpoints such as the ADAS cog, CDR-SB and MMSE.

Table 1 summarizes the composites evaluated. ADNI 1, GO, and 2 data (normal, MCI, mild AD subjects), and Phase 3 semagacestat and solanezumab placebo and treatment data (mild AD subjects) were used for the analyses. Performance of composites was evaluated by comparing the signal to noise ratio (SNR) of each of the individual composites. A total of 500 bootstraps of the original subject level dataset were performed and mixedmodel repeated measures (MMRM) analyses of postbaseline changes up to 18 (semagacestat/solanezumab) or 24 (ADNI) months were run on each bootstrapped dataset. The SNR for ADNI and semagacestat/ solanezumab placebo data was calculated by dividing the least squares mean (LSMean) change from baseline to endpoint by the standard deviation of change from baseline to endpoint. The SNR for the treatment versus placebo difference was calculated by dividing the difference in LSMean changes from baseline to endpoint by the standard deviation of the LSMean difference. Bootstrap 95\% confidence intervals were calculated to estimate the variability of the SNR estimates.

Using ADNI data:

- None of the auditory verbal learning test (AVLT) composites outperformed any of the other composites in the normal, late $\mathrm{MCI}$, and mild $\mathrm{AD}$ populations.

- For late MCI subjects (and early and late MCI combined), ADAS-Cog13+FAQ, Alzheimer's Disease Clinical Composite Score (ADCCS), Alzheimer's Disease Composite Score (ADCOMS), and Pfizer composite outperformed the ADAS-Cog11 and 13 and MMSE.

- The Prodromal Alzheimer's Disease Assessment Scale (ProADAS)+FAQ performed significantly better than ProADAS, and ADAS-Cog13+FAQ performed significantly better than ADAS-Cog13 in late MCI and the early and late $\mathrm{MCI}$ combined group. 
Table 1. Cognitive_Cognitive/Functional composites and their individual components

\begin{tabular}{|c|c|c|c|c|c|c|}
\hline Composite & ADAS-Cog items & AVLT & CDR & MMSE & FAQ & Other \\
\hline $\begin{array}{l}\text { ProADAS * } \\
\text { (Astra Zeneca) }\end{array}$ & $\begin{array}{l}\text { Word Recall } \\
\text { Delayed word recall } \\
\text { Orientation } \\
\text { Word Finding } \\
\text { Number cancellation }\end{array}$ & & & & & \\
\hline TriAD (Janssen)† & $\begin{array}{l}\text { Word Recall } \\
\text { Delayed word recall } \\
\text { Orientation }\end{array}$ & & 3 cognition boxes & & & \\
\hline ADCCS (Janssen)‡ & $\begin{array}{l}\text { Word Recall } \\
\text { Delayed word recall } \\
\text { Orientation } \\
\text { Word Recognition }\end{array}$ & & All items & & & \\
\hline ADCOMS (Eisai)§ & $\begin{array}{l}\text { Delayed word recall } \\
\text { Orientation } \\
\text { Word recognition } \\
\text { Word finding }\end{array}$ & & All items & $\begin{array}{l}\text { Orientation } \\
\text { Constructio- } \\
\text { nal-Praxis }\end{array}$ & & \\
\hline $\begin{array}{l}\text { ProADAS + FAQ / } \\
\text { iADL I I }\end{array}$ & $\begin{array}{l}\text { Word Recall } \\
\text { Delayed word recall } \\
\text { Orientation } \\
\text { Word Finding } \\
\text { Number cancellation }\end{array}$ & & & & All items & $\begin{array}{l}\text { ADCS-ADL; iADL } \\
\text { items }\end{array}$ \\
\hline $\begin{array}{l}\text { ADAS-Cog+FAQ/ } \\
\text { iADL I। }\end{array}$ & All items $\times$ & & & & All items & $\begin{array}{l}\text { ADCS-ADL; iADL } \\
\text { items }\end{array}$ \\
\hline Pfizer\{ & $\begin{array}{l}\text { Word Recall } \\
\text { Delayed Word Recall } \\
\text { Orientation }\end{array}$ & & All items & & All items & \\
\hline AVLT_Version $1 \#$ & Delayed Word Recall & & & All items & & $\begin{array}{l}\text { - Logical memory } \\
\text { IIa delayed para- } \\
\text { graph recall } \\
\text { - Digit Symbol } \\
\text { Substitution }\end{array}$ \\
\hline AVLT_Version 2\# & & Delayed Word Recall & & All items & & $\begin{array}{l}\text { - Logical memory } \\
\text { IIa delayed para- } \\
\text { graph recall } \\
\text { - Digit Symbol } \\
\text { Substitution }\end{array}$ \\
\hline AVLT_Version 3\# & & $\begin{array}{l}\text { Delayed + Imme- } \\
\text { diate recall }\end{array}$ & & All items & & $\begin{array}{l}\text { - Logical memory } \\
\text { IIa delayed para- } \\
\text { graph recall } \\
\text { - Digit Symbol } \\
\text { Substitution }\end{array}$ \\
\hline AVLT_Version 4\# & & Immediate Recall & & All items & & $\begin{array}{l}\text { - Logical memory } \\
\text { IIa delayed para- } \\
\text { graph recall } \\
\text { - Digit Symbol } \\
\text { Substitution }\end{array}$ \\
\hline AVLT_Version 5\# & & $\begin{array}{l}\text { Immediate Recall + } \\
\text { Retrieval Efficiency } \\
\text { (recognition minus } \\
\text { delayed word recall }\end{array}$ & & All items & & $\begin{array}{l}\text { - Logical memory } \\
\text { IIa delayed para- } \\
\text { graph recall } \\
\text { - Digit Symbol } \\
\text { Substitution }\end{array}$ \\
\hline
\end{tabular}

*Hannesdottir K et al (2013) Alzheimer's \& Dementia: 9(4):P461; +Raghavan N et al (2013) Alzheimer's and Dementia; \#Raghavan N (2013) Developing Composite Scores: Lessons from Janssen's Experience" CAMD pCOA Launch Meeting, April 2013; §Logovinsky V et al (2013) Alzheimer's and Parkinson's Disease Congress; I I FAQ was used in ADNI data; iADL items from the ADCS-ADL scale were used in solanezumab/semagacestat data; $\times$ ADAS-Cog13 total score was used in ADNI; ADAS-Cog14 total score was used in solanezumab/semagacestat data; \{ Huang Y. et al. (2014) Alzheimer's \& Dementia in Press; \#Computed using ADNI 1 data only; Abbreviations: ADAS-Cog, Alzheimer's Disease Assessment Scale-Cognitive Subscale; ADCCS, Alzheimer's Disease Clinical Composite Score; ADCOMS, Alzheimer's disease COMposite Score; ADNI, Alzheimer's Disease Neuroimaging Initiative; AVLT, Auditory Verbal Learning Test; CDR, Clinical Dementia Rating ScaleFAQ, Functional Assessment Questionnaire; iADL, instrumental activities of daily living; MMSE, Mini-Mental Sate Examination; ProADAS, Prodromal Alzheimer's Disease Assessment Scale; TriAD, Tri-Domain Cognitive Composite for Alzheimer's Disease. 
Using solanezumab/semagacestat data:

- In the solanezumab placebo group, ProADAS+iADL and ADCOMS outperformed ADAS-Cog11 and 14 and ADAS-Cog14+iADL outperformed ADASCog14. In the semagacestat placebo group, ADCOMS outperformed ADAS-Cog 14.

- None of the composites showed improved performance over CDR-SB in any of the placebo analyses.

- The solanezumab treatment analyses revealed that the ADAS-Cog11 and 14, ADCCS, ProADAS, ProADAS+iADL, and ADAS-Cog14+iADL were all able to detect treatment separation from placebo.

- The semagacestat treatment analyses revealed that the ADAS-Cog14, ProADAS, and ADAS-Cog14+iADL were all able to detect treatment separation from placebo.

Different composites have different abilities to detect change due to disease progression and to treatment effects. These analyses show that composites/endpoints that show a high SNR in placebo/observational data do not necessarily show a high SNR for treatment difference. Because results may differ when using different data, further study with additional data is needed to clarify these findings, further clarify the benefits of using a composite endpoint, and to identify areas for improvement. Identification and validation of a cognitive or cognitive/functional tool or composite sensitive to early changes in $\mathrm{AD}$ will be an iterative process, informed by ongoing analysis and integrating emergent data as they become available.

Conflict of interest: Authors are current or former employees and/or minor shareholders of Eli Lilly \& Company.

\section{References}

1. Zahodne LB, Manly JJ, MacKay-Brandt A, Stern Y. Cognitive declines precede and predict functional declines in aging and Alzheimer's disease. PLOS One. 2013;8(9):e73645.

2. Liu-Seifert H, Han B, Henley D, et al. Cognitive impairment precedes and predicts functional impairment in mild Alzheimer's disease. AAIC 2014a. Abstract \# F3-02-01.

3. Cano SJ, Posner HB, Moline ML, et al. The ADAS-cog in Alzheimer's disease clinical trials: psychometric evaluation of the sum and its parts. J Neurol Neurosurg Psychiatry 2010;81(12):1363-8

4. Raghavan N, Samtani MN, Farnum M, et al. The ADAS-Cog revisited: Novel Composite scales based on ADAS-Cog to improve efficiency in MCI and early AD trials. Alzheimer Dement. 2012;9(Suppl 1):S21-31.

5. Logovinsky V, Hendrix S, Perdomo C, Wang J, Satlin A. New composite score demonstrates sensitivity to disease progression and treatment effects. Presented at the AD/PD Conference on March 7, 2013.

6. Skinner J, Carvalho JO, Potter GG, et al. for the Alzheimer's Disease Neuroimaging Initiative. The Alzheimer's Disease Assessment ScaleCognitive-Plus (ADAS-Cog-Plus): an expansion of the ADAS-Cog to improve responsiveness in MCI. Brain Imaging Behav. 2012;6(4):489-501.

7. Crane PK, Carle A, Gibbons LE, et al. for the Alzheimer's Disease Neuroimaging Initiative. Development and assessment of a composite score for memory in the Alzheimer's Disease Neuroimaging Initiative (ADNI) Brain Imaging and Behavior 2012;6(4):502-516.

8. Gibbons LE, Carle AC, Mackin RS, et al. for the Alzheimer's Disease Neuroimaging Initiative. A composite score for executive functioning, validated in Alzheimer's Disease Neuroimaging Initiative (ADNI) participants with baseline mild cognitive impairment. Brain Imaging Behav. 2012;6(4):517-527. 